\title{
CONVERGENCE AND STABILITY OF A THREE-STEP ITERATIVE ALGORITHM FOR A GENERAL QUASI-VARIATIONAL INEQUALITY PROBLEM
}

\author{
K. R. KAZMI AND M. I. BHAT \\ Received 11 February 2005; Revised 10 September 2005; Accepted 13 September 2005
}

We consider a general quasi-variational inequality problem involving nonlinear, nonconvex and nondifferentiable term in uniformly smooth Banach space. Using retraction mapping and fixed point method, we study the existence of solution of general quasivariational inequality problem and discuss the convergence analysis and stability of a three-step iterative algorithm for general quasi-variational inequality problem. The theorems presented in this paper generalize, improve, and unify many previously known results in the literature.

Copyright $\odot 2006$ K. R. Kazmi and M. I. Bhat. This is an open access article distributed under the Creative Commons Attribution License, which permits unrestricted use, distribution, and reproduction in any medium, provided the original work is properly cited.

\section{Introduction}

Many problems arising in physics, mechanics, elasticity and engineering sciences can be formulated in variational inequalities involving nonlinear, nonconvex and nondifferentiable term, see for example Baiocchi and Capelo [4], Duvaut and Lions [8] and Kikuchi and Oden [15]. The proximal (resolvent) method used to study the convergence analysis of iterative algorithms for variational inclusions, see [14, 20], cannot be adopted for studying such classes of variational inequalities due to the presence of nondifferentiable term.

There are some methods, for example projection method and auxiliary principle method which can be used to study such classes of variational inequalities, see [7, 1719] and the relevent references cited therein. It is remarked that most of the work, using projection method and auxiliary principle method, has been done in the setting of Hilbert space. Recently, Alber and Yao [3] and Chen et al. [6] studied some classes of covariational inequality and co-complementarity problems in Banach spaces. Therefore, the study of other classes of variational inequalities using projection method and auxiliary principle method in the setting of Banach space remains an interesting problem. Very recently, Chidume et al. [7] studied some classes of variational inequalities involving

Hindawi Publishing Corporation

Fixed Point Theory and Applications

Volume 2006, Article ID 96012, Pages 1-16

DOI 10.1155/FPTA/2006/96012 
nonlinear, convex and nondifferentiable term, using auxiliary principle method in the setting of reflexive Banach space.

In recent years, one step and two-step iteration algorithms (including Mann Iteration and Ishikawa iteration processes as the most important cases) have been extensively studied by many authors to solve the nonlinear operator equations and variational inequality problems in Hilbert spaces and Banach spaces, see for example [3, 6, 7, 12-14, 16, 18$20,23-25,27,28]$ and the references therein. Noor $[21,22]$ introduced and analyzed three-step iterative methods to study the approximate solutions of variational inequalities (inclusions) in Hilbert spaces by using the techniques of updating the solution and the auxiliary principle. Further Xu and Noor [26] and Liu et al. [17] used three step iterative algorithms to study nonlinear operator equations and variational inequality problems, respectively. A similar idea goes back to the so called $\theta$-schemes introduced by Glowinski and Le Tallec [9] to find a zero of sum of two (or more) maximal monotone operators by using the Lagrangian multiplier. Glowinski and Le Tallec [9] used three-step iterative algorithms to find the approximate solutions of the elastoviscoplasticity problem, liquid crystal theory, and eigenvalue computation, and they showed that three-step approximations perform better numerically. Haubruge et al. [11] studied the convergence analysis of three-step iterative algorithms of Glowinski and Le Tallec [9] and applied these algorithms to obtain new splitting-type algorithms for solving variational inequalities, separable convex programming, and minimization of a sum of convex functions. They also proved that three-step iterations lead to highly parallelized algorithms under certain conditions.

It has been shown in $[11,21,22]$ that three step iterative algorithms are a natural generalization of the splitting methods for solving partial differential equations (inclusions). For applications of splitting and decomposition methods, see $[9,11,21,22]$ and the references therein. Thus one can conclude that three-step iterative algorithms play an important and significant part in solving various problems, which aries in pure and applied sciences. On the other hand there are no such three-step iterative algorithm for solving quasi-variational inequality problems in Banach spaces.

Motivated by these facts and the recent work going in this direction, we consider a general quasi-variational inequality problem (in short, GQVIP) involving nonlinear, nonconvex and nondifferentiable term, in uniformly smooth Banach space. Using sunny retraction mapping, we establish that GQVIP is equivalent to some relations. Further, using these relations, we suggest a three-step iterative algorithm for finding the approximate solution of GQVIP. Furthermore, using fixed point method, we prove the existence of unique solution of GQVIP and discuss the convergence analysis and stability of the three-step iterative algorithm. The theorems presented in this paper generalize, improve and unify the results given in $[5,12,13,18,24-27]$ and in the relevant references cited therein.

\section{Preliminaries and formulation of problem}

Throughout this paper, unless the contrary is stated, we assume that $E$ is a real uniformly smooth Banach space equipped with norm $\|\cdot\| ;\langle\cdot, \cdot\rangle$ is the dual pair between $E$ and its dual space $E^{\star} ; J: E \rightarrow E^{\star}$ be the normalized duality mapping defined by 
$\langle J(x), x\rangle=\|J(x)\|_{E^{\star}}^{2}=\|x\|_{E}^{2} \forall x \in E$ and $C C(E)$ be the family of all nonempty, closed and convex subsets of $E$. We note that if $E \equiv H$, a Hilbert space, then $J$ becomes identity mapping.

First we recall the following concepts and results which are needed in the sequel.

Definition 2.1. A single-valued mapping $g: E \rightarrow E$ is said to be

(i) $k$-strongly accretive if there exists a constant $k>0$ such that

$$
\langle g(u)-g(v), J(u-v)\rangle \geq k\|u-v\|^{2}, \quad \forall u, v \in E
$$

(ii) $\delta$-Lipschitz continuous if there exists a constant $\delta>0$ such that

$$
\|g(u)-g(v)\| \leq \delta\|u-v\|, \quad \forall u, v \in E .
$$

Definition 2.2. A mapping $N(\cdot, \cdot, \cdot): E \times E \times E \rightarrow E$ is said to be

(i) $\alpha$-strongly accretive in the first argument if there exists a constant $\alpha>0$ such that

$$
\langle N(u, \cdot, \cdot)-N(v, \cdot, \cdot), J(u-v)\rangle \geq \alpha\|u-v\|^{2}, \quad \forall u, v \in E
$$

(ii) $\beta$-Lipschitz continuous in the first argument if there exists a constant $\beta>0$ such that

$$
\|N(u, \cdot, \cdot)-N(v, \cdot, \cdot)\| \leq \beta\|u-v\|, \quad \forall u, v \in E .
$$

Definition $2.3[2,6,10]$. Let $K \subset E$ be a nonempty closed convex set. A mapping $R_{K}: E \rightarrow$ $K$ is said to be

(i) retraction if

$$
R_{K}^{2}=R_{K}
$$

(ii) nonexpansive retraction if

$$
\left\|R_{K} u-R_{K} v\right\| \leq\|u-v\|, \quad \forall u, v \in E
$$

(iii) sunny retraction if

$$
R_{K}\left(R_{K} u-t\left(u-R_{K} u\right)\right)=R_{K} u, \quad \forall u \in E, t \in R .
$$

Lemma $2.4[6,10]$. A retraction $R_{K}$ is sunny and nonexpansive if and only if

$$
\left\langle u-R_{K}(u), J\left(R_{K}(u)-v\right)\right\rangle \geq 0, \quad \forall u, v \in E .
$$


4 A general quasi-variational inequality problem

Lemma $2.5[2,6,10]$. For all $u, v \in E$, we have

(i) $\|u+v\|^{2} \leq\|u\|^{2}+2\langle v, J(u+v)\rangle$,

(ii) $\langle u-v, J u-J v\rangle \leq 2 d^{2} \rho_{E}(4\|u-v\| / d)$, where $d=\sqrt{\left(\|u\|^{2}+\|v\|^{2}\right) / 2} \rho_{E}(t)=$ $\sup \{((\|u\|+\|v\|) / 2)-1:\|u\|=1,\|v\|=t\}$ is called the modulus of smoothness of $E$.

Definition 2.6 [23]. Let $E$ be a Banach space; let $T: E \rightarrow E$ be a mapping, and let $u_{0} \in E$. Assume that $u_{n+1}=f\left(T, u_{n}\right)$ defines an iteration procedure which yields a sequence of points $\left\{u_{n}\right\}_{n=0}^{\infty} \subseteq E$. Suppose that $F(T)=\{u \in H: T(u)=u\} \neq \varnothing$ and that $\left\{u_{n}\right\}_{n=0}^{\infty} \subseteq E$ converges to some $x \in F(T)$. Let $\left\{z_{n}\right\}_{n=0}^{\infty} \subseteq E$ and $\epsilon_{n}=\left\|z_{n+1}-f\left(T, z_{n}\right)\right\|$. If $\lim _{n \rightarrow \infty} \epsilon_{n}=0$ implies $\lim _{n \rightarrow \infty} z_{n}=x$, then the iteration procedure defined by $u_{n+1}=f\left(T, u_{n}\right)$ is said to be $T$-stable or stable with respect to $T$.

Lemma 2.7 [16]. Let $\left\{a_{n}\right\},\left\{b_{n}\right\}$, and $\left\{c_{n}\right\}$ be sequences of nonnegative real numbers satisfying

$$
a_{n+1}=\left(1-\lambda_{n}\right) a_{n}+b_{n} \lambda_{n}+c_{n}, \quad \forall n \geq 0,
$$

where $\sum_{n=0}^{\infty} \lambda_{n}=\infty,\left\{\lambda_{n}\right\} \subset[0,1], \lim _{n \rightarrow \infty} b_{n}=0, \sum_{n=0}^{\infty} c_{n}<\infty$. Then $\lim _{n \rightarrow \infty} a_{n}=0$.

We remark that Lemma 2.7 is the particular case of Lemma 1 of Alber [1].

Let $N: E \times E \times E \rightarrow E$ and $g, h, A, B, C: E \rightarrow E$ be single-valued mappings and let $K$ : $E \rightarrow C C(E)$ be a set-valued mapping. We consider the following general quasi-variational inequality problem (GQVIP): Find $u \in E$ such that $g(u) \in K(u)$ and

$$
\begin{aligned}
& \langle h(g(u)), J(v-g(u))\rangle+\rho b(u, v)-\rho b(u, g(u)) \\
& \quad \geq\langle h(u), J(v-g(u))\rangle-\rho\langle N(A(u), B(u), C(u))-f, J(v-g(u))\rangle,
\end{aligned}
$$

$\forall v \in K(u)$, where $\rho>0$ is a constant; $f \in E$ and $b(\cdot, \cdot): E \times E \rightarrow \mathbb{R}$ is a nonlinear, nonconvex and nondifferentiable form satisfying the following conditions.

Condition 2.8. (i) $b(\cdot, \cdot)$ is linear in the first argument;

(ii) there exists a constant $v>0$ such that

$$
b(u, v) \leq v\|u\|\|v\|, \quad \forall u, v \in E ;
$$

(iii) $b(u, v)-b(u, w) \leq b(u, v-w), \forall u, v \in E$.

Remark 2.9. (i) Condition 2.8(i)-(ii) implies that

$$
-b(u, v) \leq v\|u\|\|v\|, \quad \forall u, v \in E .
$$

Hence, we have $|b(u, v)| \leq v\|u\|\|v\|, \forall u, v \in E$. 
(ii) Also Condition 2.8(i)-(iii) imply that

$$
|b(u, v)-b(u, w)| \leq v\|u\|\|v-w\|, \quad \forall u, v, w \in E
$$

that is, $b(u, v)$ is continuous with respect to the second argument.

2.1. Some special cases of GQVIP (2.10). (I) If $f \equiv \Theta$, where $\Theta$ is the zero element in $E ; N(u, v, w) \equiv u, \forall u, v, w \in E$, then GQVIP (2.10) reduces to the following quasivariational inequality problem: Find $u \in E$ such that $g(u) \in K(u)$ and

$$
\begin{aligned}
& \langle h(g(u)), J(v-g(u))\rangle+\rho b(u, v)-\rho b(u, g(u)) \\
& \quad \geq\langle h(u), J(v-g(u))\rangle-\rho\langle A(u), J(v-g(u))\rangle, \quad \forall v \in K(u),
\end{aligned}
$$

which appears to be new. Problem (2.14) has been studied by Zeng [27] in the setting of Hilbert space.

(II) If $f \equiv \Theta ; b \equiv 0$, a zero mapping, and $N(u, v, w) \equiv u+v, \forall u, v, w \in E$, then GQVIP (2.10) reduces to the following quasi-variational inequality problem: Find $u \in E$ such that $g(u) \in K(u)$ and

$$
\begin{aligned}
& \langle h(g(u)), J(v-g(u))\rangle \\
& \quad \geq\langle h(u), J(v-g(u))\rangle-\rho\langle(A+B)(u), J(v-g(u))\rangle, \quad \forall v \in K(u),
\end{aligned}
$$

which appears to be new. Problem (2.15) has been studied by Verma [25] in the setting of Hilbert space.

(III) If $f \equiv \Theta ; b \equiv 0$, and $N(u, v, w) \equiv u, \forall u, v, w \in E$, then GQVIP (2.10) reduces to the following quasi-variational inequality problem: Find $u \in E$ such that $g(u) \in K(u)$ and

$$
\begin{aligned}
& \langle h(g(u)), J(v-g(u))\rangle \\
& \quad \geq\langle h(u), J(v-g(u))\rangle-\rho\langle A(u), J(v-g(u))\rangle, \quad \forall v \in K(u),
\end{aligned}
$$

which is also appears to be new. Problem (2.16) has been studied by Zeng [28] in the setting of Hilbert space.

We remark that for the appropriate and suitable choices of mappings $g, h, A, B, C$, $N, b, K$, the element $f$, and the underlying space $E$, one can obtain from GQVIP (2.10) a number of known and new classes of variational and quasi-variational inequalities as special cases in the literature. 
6 A general quasi-variational inequality problem

\section{A three-step iterative algorithm}

First we prove the following important lemma.

Lemma 3.1. Let $t, \rho, \lambda$ be positive parameters with $t \leq 1$ and let Condition 2.8 be held. Then the following statements are equivalent:

(a) GQVIP (2.10) has a solution $u \in E$ with $g(u) \in K(u)$;

(b) there exists $u \in E$ such that $g(u) \in K(u)$ and

$$
\langle u-\Phi(u), J(v-g(u))\rangle \geq 0, \quad \forall v \in K(u),
$$

where the mapping $\Phi: E \rightarrow E$ is defined by

$$
\begin{aligned}
\langle\Phi(u), J(v)\rangle= & \langle u, J(v)\rangle-\langle h(g(u)), J(v)\rangle+\langle h(u), J(v)\rangle \\
& -\rho\langle N(A(u), B(u), C(u))-f, J(v)\rangle-\rho b(u, v), \quad \forall u, v \in E
\end{aligned}
$$

(c) there exists $u \in E$ such that $g(u) \in K(u)$ and

$$
g(u)=R_{K(u)}[g(u)-\lambda u+\lambda \Phi(u)]
$$

where the mapping $R_{K(u)}$ is sunny retraction from $E$ onto $K(u)$;

(d) the mapping $F: E \rightarrow E$ defined by

$$
F(u)=(1-t) u+t\left(u-g(u)+R_{K(u)}[g(u)-\lambda u+\lambda \Phi(u)]\right),
$$

for all $v \in E$ has a fixed point.

Proof. (a) $\Rightarrow$ (b). Let (a) hold, that is, $u \in E$ such that $g(u) \in K(u)$ and

$$
\begin{aligned}
& \langle h(g(u)), J(v-g(u))\rangle+\rho b(u, v)-\rho b(u, g(u)) \\
& \quad \geq\langle h(u), J(v-g(u))\rangle-\rho\langle N(A(u), B(u), C(u))-f, J(v-g(u))\rangle,
\end{aligned}
$$

which can be rewritten as

$$
\begin{aligned}
\langle u, J(v-g(u))\rangle \geq & \langle u, J(v-g(u))\rangle-\langle h(g(u)), J(v-g(u))\rangle+\langle h(u), J(v-g(u))\rangle \\
& -\rho b(u, v-g(u))-\rho\langle N(A(u), B(u), C(u))-f, J(v-g(u))\rangle .
\end{aligned}
$$

By using (3.2), the preceding inequality becomes

$$
\langle u-\Phi(u), J(v-g(u))\rangle \geq 0, \quad \forall v \in E .
$$

Hence (b) holds. 
(b) $\Rightarrow(a)$. It is immediately followed by retracing the above steps and using Condition 2.8.

Since, for $\lambda>0$,

$$
\lambda\langle u-\Phi(u), J(v-g(u))\rangle=\langle g(u)-(g(u)-\lambda u+\lambda \Phi(u)), J(v-g(u))\rangle, \quad \forall u, v \in E .
$$

Therefore, from (3.8) and Lemma 2.4, it follows the statements (b) and (c) are equivalent. Moreover, one can easily prove that for $t \in(0,1],(\mathrm{c})$ and (d) are equivalent. This completes the proof.

Based on the above lemma, we suggest the following three-step iterative algorithm for finding the approximate solution of GQVIP (2.10).

3.1. Three-step iterative algorithm (TSIA) (3.1). Let $g, h, A, B, C: E \rightarrow E ; K: E \rightarrow C C(E)$. Given $u_{0} \in E$, compute the sequence $\left\{u_{n}\right\}$ defined by the following iterative schemes:

$$
\begin{aligned}
u_{n+1} & =\left(1-\alpha_{n}\right) u_{n}+\alpha_{n}\left(v_{n}-g\left(v_{n}\right)+R_{K\left(v_{n}\right)}\left[g\left(v_{n}\right)-\lambda v_{n}+\lambda \Phi\left(v_{n}\right)\right]\right)+\alpha_{n} r_{n}, \\
v_{n} & =\left(1-\beta_{n}\right) u C+\beta_{n}\left(w_{n}-g\left(w_{n}\right)+R_{K\left(w_{n}\right)}\left[g\left(w_{n}\right)-\lambda w_{n}+\lambda \Phi\left(w_{n}\right)\right]\right)+\beta_{n} q_{n} ; \\
w_{n} & =\left(1-\gamma_{n}\right) u_{n}+\gamma_{n}\left(u_{n}-g\left(u_{n}\right)+R_{K\left(u_{n}\right)}\left[g\left(u_{n}\right)-\lambda u_{n}+\lambda \Phi\left(u_{n}\right)\right]\right)+\gamma_{n} p_{n},
\end{aligned}
$$

for $n=0,1,2,3, \ldots$, where $\Phi$ is given by

$$
\begin{aligned}
\left\langle\Phi\left(u_{n}\right), J\left(v_{n}\right)\right\rangle= & \left\langle u_{n}, J\left(v_{n}\right)\right\rangle-\left\langle h\left(g\left(u_{n}\right)\right), J\left(v_{n}\right)\right\rangle+\left\langle h\left(u_{n}\right), J\left(v_{n}\right)\right\rangle \\
& -\rho\left\langle N\left(A\left(u_{n}\right), B\left(u_{n}\right), C\left(u_{n}\right)\right)-f, J\left(v_{n}\right)\right\rangle-\rho b\left(u_{n}, v_{n}\right), \quad \forall v_{n} \in K\left(u_{n}\right) ;
\end{aligned}
$$

$\lambda>0$ is a parameter; $\left\{p_{n}\right\},\left\{q_{n}\right\},\left\{r_{n}\right\}$ are sequences of elements in $E$ introduced to take into account the possible inexact computations of the retraction points, and $\left\{\alpha_{n}\right\},\left\{\beta_{n}\right\}$, $\left\{\gamma_{n}\right\}$ are the sequences of real numbers satisfying the condition

$$
\sum_{i=0}^{\infty} \alpha_{n}=\infty, \quad 0 \leq \alpha_{n}, \beta_{n}, \gamma_{n} \leq 1, \forall n \geq 0 .
$$

\section{Existence of solution, convergence analysis, and stability}

In this section, first we establish the existence of unique solution for GQVIP (2.10) and discuss the convergence analysis of TSIA (3.1).

Theorem 4.1. Let $E$ be a uniformly smooth Banach space with $\rho_{E}(t) \leq c t^{2}$ for some constant $c>0$. Let $\lambda$ be a positive parameter; let the mappings $g, h, A, B, C: E \rightarrow E$ be q-Lipschitz continuous, $m$-Lipschitz continuous, $r$-Lipschitz continuous, s-Lipschitz continuous and $\xi$ Lipschitz continuous, respectively; let $g$ be p-strongly accretive; let the mapping $N: E \times E \times$ $E \rightarrow E$ be $\beta$-Lipschitz continuous, $\sigma$-Lipschitz continuous and $\tau$-Lipschitz continuous in the first, second and third arguments, respectively, and be $\alpha$-strongly accretive with respect to $A$ in the first argument, and let $K: E \rightarrow C C(E)$ be a set-valued mapping. Assume that for some constant $\mu>0$, 
8 A general quasi-variational inequality problem

(i)

$$
\left\|R_{K(u)}(z)-R_{K(v)}(z)\right\| \leq \mu\|u-v\|, \quad \forall u, v \in E ;
$$

(ii) $b(\cdot, \cdot): E \times E \rightarrow \mathbb{R}$ satisfy Condition 2.8 (i)-(iii);

(iii)

$$
\begin{aligned}
& \theta:=\lambda\left[k+i \rho+\sqrt{1-2 \rho \alpha+\rho^{2} d^{2}}\right] \\
& i:=v+\sigma s+\tau \xi ; \quad d^{2}:=64 c \beta^{2} r^{2},
\end{aligned}
$$

where

$$
k:=\lambda^{-1}\left[\sqrt{1-2 p+64 c q^{2}}+\mu+\sqrt{\lambda^{2}-2 \lambda p+64 c q^{2}}\right]+m(q+1) .
$$

Further assume that Condition 4.2 or Condition 4.3 below hold.

Condition 4.2. For $\rho>0$,

$$
\rho i<\lambda^{-1}-k \leq 1
$$

and one of the following conditions holds.

$$
\begin{gathered}
d>i, \\
\left|\alpha-\left(\lambda^{-1}-k\right) i\right|>\sqrt{\left(1-\left(\lambda^{-1}-k\right)^{2}\right)\left(d^{2}-i^{2}\right)} \\
\left|\rho-\frac{\alpha-\left(\lambda^{-1}-k\right) i}{d^{2}-i^{2}}\right|<\frac{\sqrt{\left(\alpha-\left(\lambda^{-1}-k\right) i\right)^{2}-\left(1-\left(\lambda^{-1}-k\right)^{2}\right)\left(d^{2}-i^{2}\right)}}{d^{2}-i^{2}} \\
d=i, \\
\rho>\left(1-\left(\lambda^{-1}-k\right)^{2}\right) / 2\left(\alpha-\left(\lambda^{-1}-k\right) i\right) ; \\
d<i, \\
\left|\rho-\frac{\left(\lambda^{-1}-k\right) i-\alpha}{i^{2}-d^{2}}\right|>\frac{\sqrt{\left(i^{2}-d^{2}\right)\left(1-\left(\lambda^{-1}-k\right)^{2}\right)+\left(\left(\lambda^{-1}-k\right) i-\alpha\right)^{2}}}{i^{2}-d^{2}} .
\end{gathered}
$$

Condition 4.3. For $\rho>0$,

$$
\max \{1, \rho i\}<\lambda^{-1}-k,
$$


and one of the following conditions holds:

$$
\begin{gathered}
d>i, \\
\left|\rho-\frac{\alpha-\left(\lambda^{-1}-k\right) i}{d^{2}-i^{2}}\right|<\frac{\sqrt{\left(\alpha-\left(\lambda^{-1}-k\right) i\right)^{2}-\left(1-\left(\lambda^{-1}-k\right)^{2}\right)\left(d^{2}-i^{2}\right)}}{d^{2}-i^{2}} \\
d=i, \\
\alpha<\left(\lambda^{-1}-k\right) i, \\
\rho<\left(\left(\lambda^{-1}-k\right)^{2}-1\right) / 2\left(\left(\lambda^{-1}-k\right) i-\alpha\right) ; \\
d<i, \\
\left|\left(\lambda^{-1}-k\right) i-\alpha\right|>\sqrt{\left(\left(\lambda^{-1}-k\right)^{2}-1\right)\left(d^{2}-i^{2}\right)} \\
\left|\rho-\frac{\left(\lambda^{-1}-k\right) i-\alpha}{i^{2}-d^{2}}\right|>\frac{\sqrt{\left(i^{2}-d^{2}\right)\left(1-\left(\lambda^{-1}-k\right)^{2}\right)+\left(\left(\lambda^{-1}-k\right) i-\alpha\right)^{2}}}{i^{2}-d^{2}}
\end{gathered}
$$

Then GQVIP (2.10) has a unique solution $u \in E$. Further, the sequence $\left\{u_{n}\right\}$ generated by TSIA (3.1), converges strongly to $u$ provided that

$$
\lim _{n \rightarrow \infty} \beta_{n} \gamma_{n}\left\|p_{n}\right\|=\lim _{n \rightarrow \infty} \beta_{n}\left\|q_{n}\right\|=\lim _{n \rightarrow \infty}\left\|r_{n}\right\|=0
$$

Proof. From (3.4), (4.1) and Lemma 2.4, we estimate $\|F(u)-F(v)\|$ :

$$
\begin{aligned}
\|F(u)-F(v)\|= & \|(1-t) u+t\left(u-g(u)+R_{K(u)}[g(u)-\lambda u+\lambda \Phi(u)]\right) \\
& +(1-t) v+t\left(v-g(v)+R_{K(v)}[g(v)-\lambda v+\lambda \Phi(v)]\right) \| \\
\leq & (1-t)\|u-v\|+t\|u-v-(g(u)-g(v))\| \\
& +t\left\|R_{K(u)}[g(u)-\lambda u+\lambda \Phi(u)]-R_{K(u)}[g(v)-\lambda v+\lambda \Phi(v)]\right\| \\
& +t\left\|R_{K(u)}[g(v)-\lambda v+\lambda \Phi(v)]-R_{K(v)}[g(v)-\lambda v+\lambda \Phi(v)]\right\| \\
\leq & (1-t)\|u-v\|+t\|u-v-(g(u)-g(v))\| \\
& +t\|g(u)-g(v)-\lambda(u-v)+\lambda(\Phi(u)-\Phi(v))\|+t \mu\|u-v\| \\
\leq & (1-t)\|u-v\|+t\|u-v-(g(u)-g(v))\| \\
& +t\|g(u)-g(v)-\lambda(u-v)\|+t \lambda\|\Phi(u)-\Phi(v)\|+t \mu\|u-v\| .
\end{aligned}
$$


10 A general quasi-variational inequality problem

Now since $g$ is $p$-strongly accretive and $q$-Lipschitz continuous then by using Lemma 2.5 , we have

$$
\begin{aligned}
\| u-v- & (g(u)-g(v)) \|^{2} \\
\leq & \|u-v\|^{2}-2\langle g(u)-g(v), J(u-v-(g(u)-g(v)))\rangle \\
= & \|u-v\|^{2}-2\langle g(u)-g(v), J(u-v)\rangle \\
& +2\langle g(u)-g(v), J(u-v)-J(u-v-(g(u)-g(v)))\rangle \\
\leq & \left(1-2 p+64 c q^{2}\right)\|u-v\|^{2},
\end{aligned}
$$

and similarly, we have

$$
\|g(u)-g(v)-\lambda(u-v)\| \leq \sqrt{\lambda^{2}-2 \lambda p+64 c q^{2}}\|u-v\|
$$

Now, using (2.14), Condition 2.8(i), and Remark 2.9(ii), we have

$$
\begin{aligned}
\| \Phi(u)- & \Phi(v) \|^{2} \\
= & |\langle\Phi(u)-\Phi(v), J(\Phi(u)-\Phi(v))\rangle| \\
= & \mid\langle u-v, J(\Phi(u)-\Phi(v))\rangle-\rho b(u, \Phi(u)-\Phi(v))+\rho b(v, \Phi(u)-\Phi(v)) \\
& -\langle h(g(u))-h(g(v)), J(\Phi(u)-\Phi(v))\rangle+\langle h(u)-h(v), J(\Phi(u)-\Phi(v))\rangle \\
& -\rho\langle N(A(u), B(u), C(u))-N(A(v), B(v), C(v)), J(\Phi(u)-\Phi(v))\rangle \mid \\
\leq & \mid\langle u-v-(h(g(u))-h(g(v)))+(h(u)-h(v)) \\
& -\rho[N(A(u), B(u), C(u))-N(A(v), B(v), C(v))], J(\Phi(u)-\Phi(v))\rangle \mid \\
& +\rho|b(u-v, \Phi(u)-\Phi(v))| \\
\leq & (\|u-v-\rho[N(A(u), B(u), C(u))-N(A(v), B(v), C(v))]\| \\
& +\|h(g(u))-h(g(v))\|+\|h(u)-h(v)\|)\|\Phi(u)-\Phi(v)\| \\
& +\rho|b(u-v, \Phi(u)-\Phi(v))| \\
\leq & (\|u-v-\rho[N(A(u), B(u), C(u))-N(A(v), B(v), C(v))]\| \\
& +\|h(g(u))-h(g(v))\|+\|h(u)-h(v)\|+\rho v\|u-v\|)\|\Phi(u)-\Phi(v)\| .
\end{aligned}
$$


Now, since $g$ and $h$ are $q$-Lipschitz continuous and $m$-Lipschitz continuous, respectively, the preceding inequality reduces to

$$
\begin{aligned}
\|\Phi(u)-\Phi(v)\| \leq & \|u-v-\rho[N(A(u), B(u), C(u))-N(A(v), B(v), C(v))]\| \\
& +(m(q+1)+\rho v)\|u-v\| .
\end{aligned}
$$

Next, we have the following estimate:

$$
\begin{aligned}
\| u-v- & \rho[N(A(u), B(u), C(u))-N(A(v), B(v), C(v))] \| \\
\leq & \|u-v-\rho[N(A(u), B(u), C(u))-N(A(v), B(u), C(u))]\| \\
& +\rho\|N(A(v), B(u), C(u))-N(A(v), B(v), C(u))\| \\
& +\rho\|N(A(v), B(v), C(u))-N(A(v), B(v), C(v))\| .
\end{aligned}
$$

Since $N$ is $\alpha$-strongly accretive with respect to $A$ in the first argument, and $N$ is $\beta$ Lipschitz continuous, $\sigma$-Lipschitz continuous and $\tau$-Lipschitz continuous with respect to the first, second and third arguments, respectively, we can easily obtain the following estimates:

$$
\begin{aligned}
& \|u-v-\rho[N(A(u), B(u), C(u))-N(A(v), B(u), C(u))]\| \\
& \quad \leq \sqrt{1-2 \rho \alpha+64 c \rho^{2} \beta^{2} r^{2}}\|u-v\| ; \\
& \|N(A(v), B(u), C(u))-N(A(v), B(v), C(u))\| \leq \sigma s\|u-v\| ; \\
& \|N(A(v), B(v), C(u))-N(A(v), B(v), C(v))\| \leq \tau \xi\|u-v\| .
\end{aligned}
$$

Combining (4.18)-(4.21), we have

$$
\begin{gathered}
\|u-v-\rho[N(A(u), B(u), C(u))-N(A(v), B(v), C(v))]\| \\
\leq\left(\sqrt{1-2 \rho \alpha+64 c \rho^{2} \beta^{2} r^{2}}+\rho(\sigma s+\tau \xi)\right)\|u-v\| .
\end{gathered}
$$

From (4.17) and (4.22), we have

$$
\|\Phi(u)-\Phi(v)\| \leq\left(\sqrt{1-2 \rho \alpha+64 c \rho^{2} \beta^{2} r^{2}}+\rho(\nu+\sigma s+\tau \xi)+m(q+1)\right)\|u-v\| .
$$

From (4.13)-(4.15) and (4.23), we have

$$
\begin{gathered}
\|F(u)-F(v)\| \leq\left(1-t+t\left[\sqrt{1-2 p+64 c q^{2}}+\sqrt{\lambda^{2}-2 \lambda p+64 c q^{2}}+\mu+\lambda m(q+1)\right.\right. \\
\left.\left.+\lambda \rho(\nu+\sigma s+\tau \xi)+\lambda \sqrt{1-2 \rho \alpha+64 c \rho^{2} \beta^{2} r^{2}}\right]\right)\|u-v\| \\
=(1-t(1-\theta))\|u-v\| .
\end{gathered}
$$


Now,

$$
\begin{aligned}
\theta<1 & \Longleftrightarrow \sqrt{1-2 \rho \alpha+\rho^{2} d^{2}}<\left(\lambda^{-1}-k\right)-i \rho \\
& \Longleftrightarrow \rho^{2}\left(d^{2}-i^{2}\right)-2 \rho\left(\alpha-\left(\lambda^{-1}-k\right) i\right)<\left(\lambda^{-1}-k\right)^{2}-1
\end{aligned}
$$

From either Condition 4.2 or Condition 4.3, and (4.25), it follows that $\theta<1$. Since $t \in(0,1]$, it follows from (4.24) that $F$ is a contraction mapping. Therefore, by Banach contraction principle, $F$ has a unique fixed point $u$ in $E$. Thus it follows from Lemma 3.1 that GQVIP (2.10) has a unique solution $u$ in $E$. Further, we observe that $u$ satisfies

$$
\begin{aligned}
& u=\left(1-\alpha_{n}\right) u+\alpha_{n}\left(u-g(u)+R_{K(u)}[g(u)-\lambda u+\lambda \Phi(u)]\right) ; \\
& u=\left(1-\beta_{n}\right) u+\beta_{n}\left(u-g(u)+R_{K(u)}[g(u)-\lambda u+\lambda \Phi(u)]\right) ; \\
& u=\left(1-\gamma_{n}\right) u+\gamma_{n}\left(u-g(u)+R_{K(u)}[g(u)-\lambda u+\lambda \Phi(u)]\right),
\end{aligned}
$$

for all $n=0,1,2,3, \ldots$.

Using (3.2), (4.26) and repeating the above arguments, we obtain

$$
\left\|\Phi\left(u_{n}\right)-\Phi(u)\right\| \leq\left(\sqrt{1-2 \rho \alpha+\rho^{2} d^{2}}+\rho i+m(q+1)\right)\left\|u_{n}-u\right\| .
$$

It follows from (3.10), (4.26), (4.27), and Lemma 2.4 that

$$
\begin{aligned}
\left\|w_{n}-u\right\| \leq & \left(1-\gamma_{n}\right)\left\|u_{n}-u\right\|+\gamma_{n}\left\|u_{n}-u-\left(g\left(u_{n}\right)-g(u)\right)\right\|+\gamma_{n}\left\|p_{n}\right\| \\
& +\gamma_{n}\left\|R_{K\left(u_{n}\right)}\left(g\left(u_{n}\right)-\lambda u_{n}+\lambda \Phi\left(u_{n}\right)\right)-R_{K(u)}(g(u)-\lambda u+\lambda \Phi(u))\right\| \\
\leq & \left(1-\gamma_{n}+\gamma_{n} \sqrt{1-2 p+64 c \rho^{2} q^{2}}+\gamma_{n} \mu\right)\left\|u_{n}-u\right\|+\gamma_{n}\left\|p_{n}\right\| \\
& +\left\|g\left(u_{n}\right)-g(u)-\lambda\left(u_{n}-u\right)\right\|+\gamma_{n} \lambda\left\|\Phi\left(u_{n}\right)-\Phi(u)\right\| \\
\leq & \left(1-(1-\theta) \gamma_{n}\right)\left\|u_{n}-u\right\|+\gamma_{n}\left\|p_{n}\right\| \\
\leq & \left\|u_{n}-u\right\|+\gamma_{n}\left\|p_{n}\right\|, \quad \text { since }\left(1-(1-\theta) \gamma_{n}\right) \leq 1 .
\end{aligned}
$$

By using similar arguments as above and (4.27), we have the following estimates:

$$
\begin{aligned}
\left\|v_{n}-u\right\| \leq & \left(1-\beta_{n}\right)\left\|u_{n}-u\right\|+\beta_{n}\left\|w_{n}-u-\left(g\left(w_{n}\right)-g(u)\right)\right\|+\beta_{n}\left\|q_{n}\right\| \\
& +\beta_{n}\left\|R_{K\left(w_{n}\right)}\left(g\left(w_{n}\right)-\lambda w_{n}+\lambda \Phi\left(w_{n}\right)\right)-R_{K(u)}(g(u)-\lambda u+\lambda \Phi(u))\right\| \\
\leq & \left(1-\beta_{n}\right)\left\|u_{n}-u\right\|+\theta \beta_{n}\left\|w_{n}-u\right\|+\beta_{n}\left\|q_{n}\right\| \\
\leq & \left(1-(1-\theta) \beta_{n}\right)\left\|u_{n}-u\right\|+\theta \beta_{n} \gamma_{n}\left\|p_{n}\right\|+\beta_{n}\left\|q_{n}\right\| \\
\leq & \left\|u_{n}-u\right\|+\theta \beta_{n} \gamma_{n}\left\|p_{n}\right\|+\beta_{n}\left\|q_{n}\right\|, \quad \text { since }\left(1-(1-\theta) \beta_{n}\right) \leq 1,
\end{aligned}
$$


and by using (4.29), we have

$$
\begin{aligned}
\left\|u_{n+1}-u\right\| \leq & \left(1-\alpha_{n}\right)\left\|u_{n}-u\right\|+\alpha_{n}\left\|v_{n}-u-\left(g\left(v_{n}\right)-g(u)\right)\right\|+\alpha_{n}\left\|r_{n}\right\| \\
& +\alpha_{n}\left\|R_{K\left(v_{n}\right)}\left(g\left(v_{n}\right)-\lambda v_{n}+\lambda \Phi\left(v_{n}\right)\right)-R_{K(u)}(g(u)-\lambda u+\lambda \Phi(u))\right\| \\
\leq & \left(1-\alpha_{n}\right)\left\|u_{n}-u\right\|+\theta \alpha_{n}\left\|v_{n}-u\right\|+\alpha_{n}\left\|r_{n}\right\| \\
\leq & \left(1-(1-\theta) \alpha_{n}\right)\left\|u_{n}-u\right\|+\alpha_{n}\left(\theta^{2} \beta_{n} \gamma_{n}\left\|p_{n}\right\|+\theta \beta_{n}\left\|q_{n}\right\|+\left\|r_{n}\right\|\right) .
\end{aligned}
$$

Setting

$$
\begin{gathered}
a_{n}=\left\|u_{n}-u\right\| ; \quad \lambda_{n}=(1-\theta) \alpha_{n} ; \\
b_{n}=(1-\theta)^{-1}\left(\theta^{2} \beta_{n} \gamma_{n}\left\|p_{n}\right\|+\theta \beta_{n}\left\|q_{n}\right\|+\left\|r_{n}\right\|\right) ; \\
c_{n}=0, \quad \forall n .
\end{gathered}
$$

It follows from Lemma 2.7, (3.12), (4.12), and (4.30) that $a_{n} \rightarrow 0$ as $n \rightarrow \infty$, that is, $u_{n} \rightarrow u$ as $n \rightarrow \infty$. This completes the proof.

Finally, we discuss the stability of TSIA (3.1).

Corollary 4.4. Let $E, g, h, A, B, C, N$, and $K$ be same as in Theorem 4.1. Let the assumptions (4.1)-(4.3), (4.12), and either Condition 4.2 or Condition 4.3 of Theorem 4.1 hold. Let $\left\{z_{n}\right\}$ be any sequence in $E$ and let $\left\{\delta_{n}\right\} \subseteq[0, \infty)$ be defined as

$$
\delta_{n}=\left\|z_{n+1}-\left(1-\alpha_{n}\right) z_{n}-\alpha_{n}\left(y_{n}-g\left(y_{n}\right)+R_{K\left(y_{n}\right)}\left[g\left(y_{n}\right)-\lambda y_{n}+\lambda \Phi\left(y_{n}\right)\right]\right)-\alpha_{n} r_{n}\right\|,
$$

where

$$
\begin{aligned}
& y_{n}=\left(1-\beta_{n}\right) z_{n}+\beta_{n}\left(x_{n}-g\left(x_{n}\right)+R_{K\left(x_{n}\right)}\left[g\left(x_{n}\right)-\lambda x_{n}+\lambda \Phi\left(x_{n}\right)\right]\right)+\beta_{n} q_{n} ; \\
& x_{n}=\left(1-\gamma_{n}\right) z_{n}+\gamma_{n}\left(z_{n}-g\left(z_{n}\right)+R_{K\left(z_{n}\right)}\left[g\left(z_{n}\right)-\lambda z_{n}+\lambda \Phi\left(z_{n}\right)\right]\right)+\gamma_{n} p_{n},
\end{aligned}
$$

for all $n=0,1,2,3, \ldots$, and $\Phi$ is defined by (3.11).

If there exists $\omega>0$ such that

$$
\alpha_{n} \geq \omega, \quad \forall n \geq 0,
$$

then

$$
\lim _{n \rightarrow \infty} z_{n}=u, \quad \text { iff } \lim _{n \rightarrow \infty} \delta_{n}=0 .
$$

Proof. Using the arguments used in Theorem 4.1 for obtaining (4.28) and (4.29), we have

$$
\begin{aligned}
& \left\|x_{n}-u\right\| \leq\left(1-(1-\theta) \gamma_{n}\right)\left\|z_{n}-u\right\|+\gamma_{n}\left\|p_{n}\right\| \leq\left\|z_{n}-u\right\|+\gamma_{n}\left\|p_{n}\right\| ; \\
& \left\|y_{n}-u\right\| \leq\left(1-(1-\theta) \beta_{n}\right)\left\|z_{n}-u\right\|+\theta \beta_{n} \gamma_{n}\left\|p_{n}\right\|+\beta_{n}\left\|q_{n}\right\|,
\end{aligned}
$$


14 A general quasi-variational inequality problem

and

$$
\begin{aligned}
\|(1- & \left.\alpha_{n}\right) z_{n}+\alpha_{n}\left(y_{n}-g\left(y_{n}\right)+R_{K\left(y_{n}\right)}\left[g\left(y_{n}\right)-\lambda y_{n}+\lambda \Phi\left(y_{n}\right)\right]\right)+\alpha_{n} r_{n}-u \| \\
& \leq\left(1-\alpha_{n}\right)\left\|z_{n}-u\right\|+\theta \alpha_{n}\left\|y_{n}-u\right\|+\alpha_{n}\left\|r_{n}\right\| \\
& \leq\left(1-(1-\theta) \alpha_{n}\right)\left\|z_{n}-u\right\|+\alpha_{n}\left(\theta^{2} \beta_{n} \gamma_{n}\left\|p_{n}\right\|+\theta \beta_{n}\left\|q_{n}\right\|+\left\|r_{n}\right\|\right) .
\end{aligned}
$$

Suppose that $\lim _{n \rightarrow \infty} \delta_{n}=0$.

Using (4.34) and (4.37), we estimate that

$$
\begin{aligned}
\left\|z_{n+1}-u\right\| \leq & \left\|\left(1-\alpha_{n}\right) z_{n}+\alpha_{n}\left(y_{n}-g\left(y_{n}\right)+R_{K\left(y_{n}\right)}\left[g\left(y_{n}\right)-\lambda y_{n}+\lambda \Phi\left(y_{n}\right)\right]\right)+\alpha_{n} r_{n}-u\right\| \\
& +\left\|z_{n+1}-\left(1-\alpha_{n}\right) z_{n}-\alpha_{n}\left(y_{n}-g\left(y_{n}\right)+R_{K\left(y_{n}\right)}\left[g\left(y_{n}\right)-\lambda y_{n}+\lambda \Phi\left(y_{n}\right)\right]\right)-\alpha_{n} r_{n}\right\| \\
\leq & \left(1-(1-\theta) \alpha_{n}\right)\left\|z_{n}-u\right\|+\alpha_{n}\left(\theta^{2} \beta_{n} \gamma_{n}\left\|p_{n}\right\|+\theta \beta_{n}\left\|q_{n}\right\|+\left\|r_{n}\right\|+\alpha_{n}^{-1} \delta_{n}\right) \\
\leq & \left(1-(1-\theta) \alpha_{n}\right)\left\|z_{n}-u\right\|+\alpha_{n}\left(\theta^{2} \beta_{n} \gamma_{n}\left\|p_{n}\right\|+\theta \beta_{n}\left\|q_{n}\right\|+\left\|r_{n}\right\|+\omega^{-1} \delta_{n}\right) .
\end{aligned}
$$

Setting

$$
\begin{gathered}
a_{n}=\left\|z_{n}-u\right\| ; \quad \lambda_{n}=(1-\theta) \alpha_{n} ; \\
b_{n}=(1-\theta)^{-1}\left(\theta^{2} \beta_{n} \gamma_{n}\left\|p_{n}\right\|+\theta \beta_{n}\left\|q_{n}\right\|+\left\|r_{n}\right\|+\omega^{-1} \delta_{n}\right) ; \\
c_{n}=0, \quad \forall n .
\end{gathered}
$$

It follows from Lemma 2.7, (3.12), (4.12), and (4.38) that $a_{n} \rightarrow 0$ as $n \rightarrow \infty$, that is, $z_{n} \rightarrow u$ as $n \rightarrow \infty$.

Conversely, suppose that $\lim _{n \rightarrow \infty} z_{n}=u$. Then (4.38), (4.12) and (4.34) ensure that

$$
\begin{aligned}
\delta_{n} \leq & \left\|z_{n+1}-u\right\| \\
& +\left\|\left(1-\alpha_{n}\right) z_{n}+\alpha_{n}\left(y_{n}-g\left(y_{n}\right)+R_{K\left(y_{n}\right)}\left[g\left(y_{n}\right)-\lambda y_{n}+\lambda \Phi\left(y_{n}\right)\right]\right)+\alpha_{n} r_{n}-u\right\| \\
\leq & \left(1-(1-\theta) \alpha_{n}\right)\left\|z_{n}-u\right\|+\alpha_{n}\left(\beta_{n} \gamma_{n}\left\|p_{n}\right\|+\beta_{n}\left\|q_{n}\right\|+\left\|r_{n}\right\|\right) \\
\leq & \left\|z_{n+1}-u\right\|+(1-(1-\theta) \omega)\left\|z_{n}-u\right\|+\theta^{2} \beta_{n} \gamma_{n}\left\|p_{n}\right\|+\theta \beta_{n}\left\|q_{n}\right\|+\left\|r_{n}\right\| \\
& \longrightarrow 0 \text { as } n \longrightarrow \infty .
\end{aligned}
$$

Hence, $\delta_{n} \rightarrow 0$ as $n \rightarrow \infty$. This completes the proof.

\section{Acknowledgment}

The authors would like to express their thanks to the referee for his valuable comments and suggestions. 


\section{References}

[1] Ya. I. Alber, The solution of equations and variational inequalities with maximal monotone operators, Soviet Mathematics Doklady 20 (1979), 871-876 (Russian).

[2] __ Metric and generalized projection operators in Banach spaces: properties and applications, Theory and Applications of Nonlinear Operators of Accretive and Monotone Type (A. G. Kartsatos, ed.), Lecture Notes in Pure and Applied Mathematics, vol. 178, Dekker, New York, 1996, pp. 15-50.

[3] Ya. I. Alber and J.-C. Yao, Algorithm for generalized multi-valued co-variational inequalities in Banach spaces, Functional Differential Equations 7 (2000), no. 1-2, 5-13 (2001).

[4] C. Baiocchi and A. Capelo, Variational and Quasivariational Inequalities. Applications to Free Boundary Problems, A Wiley-Interscience Publication, John Wiley \& Sons, New York, 1984.

[5] R. K. Bose, On a general nonlinear variational inequality, Bulletin of the Australian Mathematical Society 42 (1990), no. 3, 399-406.

[6] J.-Y. Chen, N.-C. Wong, and J.-C. Yao, Algorithm for generalized co-complementarity problems in Banach spaces, Computers \& Mathematics with Applications 43 (2002), no. 1-2, 49-54.

[7] C. E. Chidume, K. R. Kazmi, and H. Zegeye, General auxiliary problem and algorithm for a generalized multi-valued variational-like inequality problem in reflexive Banach spaces, Applicable Analysis 82 (2003), no. 12, 1099-1109.

[8] G. Duvaut and J.-L. Lions, Inequalities in Mechanics and Physics, Grundlehren der mathematischen Wissenschaften, vol. 219, Springer, Berlin, 1976.

[9] R. Glowinski and P. Le Tallec, Augmented Lagrangian and Operator-Splitting Methods in Nonlinear Mechanics, SIAM Studies in Applied Mathematics, vol. 9, SIAM, Pennsylvania, 1989.

[10] K. Goebel and S. Reich, Uniform Convexity, Hyperbolic Geometry, and Nonexpansive Mappings, Monographs and Textbooks in Pure and Applied Mathematics, vol. 83, Marcel Dekker, New York, 1984.

[11] S. Haubruge, V. H. Nguyen, and J. J. Strodiot, Convergence analysis and applications of the Glowinski-Le Tallec splitting method for finding a zero of the sum of two maximal monotone operators, Journal of Optimization Theory and Applications 97 (1998), no. 3, 645-673.

[12] K. R. Kazmi, On a class of nonlinear quasivariational inequalities, New Zealand Journal of Mathematics 24 (1995), no. 2, 17-23.

[13] _ Generalized quasivariational inequality problems in Hilbert spaces, Sichuan Shifan Daxue Xuebao Ziran Kexue Ban 20 (1997), no. 3, 1-5.

[14] K. R. Kazmi and M. I. Bhat, Convergence and stability of iterative algorithms of generalized setvalued variational-like inclusions in Banach spaces, Applied Mathematics and Computation 166 (2005), no. 1, 164-180.

[15] N. Kikuchi and J. T. Oden, Contact Problems in Elasticity: A Study of Variational Inequalities and Finite Element Methods, SIAM Studies in Applied Mathematics, vol. 8, SIAM, Pennsylvania, 1988.

[16] L. S. Liu, Ishikawa and Mann iterative process with errors for nonlinear strongly accretive mappings in Banach spaces, Journal of Mathematical Analysis and Applications 194 (1995), no. 1, 114125.

[17] Z. Liu, J. S. Ume, and S. M. Kang, General strongly nonlinear quasivariational inequalities with relaxed Lipschitz and relaxed monotone mappings, Journal of Optimization Theory and Applications 114 (2002), no. 3, 639-656.

[18] M. A. Noor, An iterative scheme for a class of quasivariational inequalities, Journal of Mathematical Analysis and Applications 110 (1985), no. 2, 463-468.

[19] _ Auxiliary principle for generalized mixed variational-like inequalities, Journal of Mathematical Analysis and Applications 215 (1997), no. 1, 75-85.

[20] - Generalized set-valued variational inclusions and resolvent equations, Journal of Mathematical Analysis and Applications 228 (1998), no. 1, 206-220. 


\section{A general quasi-variational inequality problem}

[21]_ New approximation schemes for general variational inequalities, Journal of Mathematical Analysis and Applications 251 (2000), no. 1, 217-229.

[22] _ Three-step approximation schemes for multivalued quasi variational inclusions, Nonlinear Functional Analysis and Applications 6 (2001), no. 3, 383-394.

[23] M. O. Osilike, Stability results for the Ishikawa fixed point iteration procedure, Indian Journal of Pure and Applied Mathematics 26 (1995), no. 10, 937-945.

[24] A. H. Siddiqi and Q. H. Ansari, An algorithm for a class of quasivariational inequalities, Journal of Mathematical Analysis and Applications 145 (1990), no. 2, 413-418.

[25] R. U. Verma, The solvability of a class of generalized nonlinear variational inequalities based on an iterative algorithm, Applied Mathematics Letters 12 (1999), no. 4, 51-53.

[26] B. Xu and M. A. Noor, Fixed-point iterations for asymptotically nonexpansive mappings in Banach spaces, Journal of Mathematical Analysis and Applications 267 (2002), no. 2, 444-453.

[27] L.-C. Zeng, A note on general algorithm for variational inequalities, Journal of Mathematical Analysis and Applications 223 (1998), no. 1, 354-363.

[28]_, On a general projection algorithm for variational inequalities, Journal of Optimization Theory and Applications 97 (1998), no. 1, 229-235.

K. R. Kazmi: Department of Mathematics, Aligarh Muslim University, Aligarh 202002, India E-mail address: krkazmi@gmail.com

M. I. Bhat: Department of Mathematics, Aligarh Muslim University, Aligarh 202002, India Current address: Department of Applied Mathematics, Baba Ghulam Shah Badshah University, Rajouri, Jammu and Kashmir, India

E-mail address: iqbal92@gmail.com 